https://helda.helsinki.fi

\title{
Cosmological perturbations in restricted $f(R)$ gravity
}

\section{Chaichian, M.}

2017-04-06

Chaichian , M , Ghalee , A \& Kluson , J 2017 , ' Cosmological perturbations in restricted f(R) gravity ' , Physical Review D , vol. 95 , no. 8 , 084009 . https://doi.org/10.1103/PhysRevD.95.084009

http://hdl.handle.net/10138/217914

https://doi.org/10.1103/PhysRevD.95.084009

cc_by

publishedVersion

Downloaded from Helda, University of Helsinki institutional repository.

This is an electronic reprint of the original article.

This reprint may differ from the original in pagination and typographic detail.

Please cite the original version. 
PHYSICAL REVIEW D 95, 084009 (2017)

\title{
Cosmological perturbations in restricted $f(R)$ gravity
}

\author{
M. Chaichian, ${ }^{1, *}$ A. Ghalee, ${ }^{2, \dagger}$ and J. Klusoñ ${ }^{3, \$}$ \\ ${ }^{1}$ Department of Physics, University of Helsinki, P.O. Box 64, FI-00014 Helsinki, Finland \\ ${ }^{2}$ Department of Physics, Tafresh University, Tafresh 39518 79611, Iran \\ ${ }^{3}$ Department of Theoretical Physics and Astrophysics, Faculty of Science, Masaryk University, \\ Kotlářská 2, 61137 Brno, Czech Republic \\ (Received 9 September 2016; published 6 April 2017)
}

\begin{abstract}
We investigate the metric perturbations of the restricted $f(R)$ theory of gravity in the cosmological context and explore the phenomenological implications of this model. We show that it is possible to construct a restricted model of gravity, in which the background equations are the same as the equations of motion which are derived from the Einstein-Hilbert action with the cosmological constant term. We argue that the deviation from the Einstein-Hilbert model emerges in the perturbed equations, for which we have a nonvanishing anisotropic stress. Further, with the help of the results of Planck data for the modified gravity, we obtain constraints on the parameters of the model.
\end{abstract}

DOI: 10.1103/PhysRevD.95.084009

\section{INTRODUCTION}

Observations of the cosmic microwave background from Plank 2015 data show that there exists an inflationary phase for the early Universe [1]. It has been argued that the Starobinsky model [2], in which the Einstein-Hilbert (EH) action for gravity is modified by adding the square of the Ricci scalar in action, is in agreement with observations [1].

Also, various observational data show that the Universe is currently in the accelerated expansion phase [3]. Although the late-time acceleration in the expansion of the Universe can be explained by adding the cosmological constant (CC) in the EH action, the CC term confronts us with the CC problem [4].

Since cosmology of the early Universe can be explained by modified gravity, it is natural to use the modified gravities to explain the late-time cosmology and avoid the CC problem. One way to construct modified gravity is to use manifestly covariant modifications of the $\mathrm{EH}$ action. For example, one of the candidates to produce the accelerated expansion phase is $f(R)$ gravity which is described by the action ${ }^{1}$

$S_{f(R)}=\int d^{4} x \sqrt{-g}\left[\frac{M_{P}^{2} R}{2}+\eta M_{P}^{4} f\left(\frac{R}{M_{P}^{2}}\right)\right]+S_{\text {Matter }}$,

where $M_{P}^{2}$ is the reduced Planck mass and $\eta$ is a dimensionless constant. This action is invariant under the full four-dimensional diffeomorphism by construction.

On the other hand, the existence of cosmic microwave background radiation shows that the Universe has

\footnotetext{
*masud.chaichian@helsinki.fi

†ghalee@ut.ac.ir

*klu@physics.muni.cz

${ }^{1}$ For a review and extensive list of references, see Ref. [5].
}

a preferred coordinate, which is described by the Friedmann-Robertson-Walker (FRW) metric. Regarding the last point, some phenomenological models have been proposed in which the full four-dimensional diffeomorphism is broken [6,7].

Recently, a new model of restricted $f(R)$ gravity has been proposed in Ref. [8]. This proposal is based on the idea of a mild breaking of the diffeomorphism invariance of the four-dimensional $f(R)$ gravity in very specific way. Explicitly, we use well known $3+1$ decomposition of the scalar curvature $[9,10]$, and we change the coefficient in front of the derivative term which, in the standard general relativity, is a total derivative and can be ignored. In the case of $f(R)$ gravity, the situation is completely different, and this term has crucial role in the formulation of this theory. In the case of the restricted $f(R)$ gravity, the modification of this term is even more crucial for the theoretical consistency of the theory as we showed in our previous paper [8] and as we review in Sec. II. On the other hand, the existence of the new parameter that controls the breaking of the full diffeomorphism invariance is crucial for the phenomenological applications of this theory. Explicitly, we also analyzed cosmological solutions of the restricted $f(R)$ gravity, and we found new solutions of which the properties depend on the value of the parameter $\Upsilon$. It is important to emphasize that some of these solutions cannot be found in the diffeomorphisminvariant $f(R)$ gravity.

Due to the fact that there are new cosmological solutions, it is natural to investigate them in more detail. The aim of this paper is to focus on the analysis of the cosmological fluctuations of the restricted $f(R)$ gravity. For the first step, we determine the background equations from the restricted $f(R)$-gravity action when we focus on a time-dependent ansatz. Then, we analyze the fluctuations above this background. Due to the fact that the scalar, vector and 


\section{CHAICHIAN, A. GHALEE, and J. KLUSOŇ}

tensor fluctuations decouple at the quadratic order, ${ }^{2}$ we can analyze each kind of fluctuations separately. We show that the restricted $f(R)$ gravity differs from the standard $f(R)$ gravity in the scalar sector, while the vector and tensor sectors have the same properties as in the case of diffeomorphism-invariant $f(R)$ gravity. More precisely, we show that with a suitable choice of parameters the restricted $f(R)$ gravity allows us to explain recent observation data that predict the possibility of the existence of a nonzero anisotropic stress (see for example Ref. [12]).

This paper is organized as follows. In Sec. II, we review theoretical grounds of the restricted $f(R)$ theory of gravity. Then, in Sec. III, we briefly review the equations of motion of the restricted $f(R)$ gravity evaluated on the timedependent cosmological solution. In Sec. IV, we analyze the fluctuations above this background, and in Sec. V, we discuss our results with relation to the recent phenomenological observations and obtain some constraints on the parameters in cases that the model can be solved analytically. Finally, in Sec. VI, we outline our results.

\section{REVIEW OF RESTRICTED $\boldsymbol{f}(\boldsymbol{R})$ THEORY OF GRAVITY}

In this section, we review basic facts about restricted $f(R)$ theory of gravity as was formulated in Ref. [8]. As we argued in the Introduction, restricted $f(R)$ gravity is based on the $3+1$ formulation of gravity. For that reason, we introduce the $3+1$ decomposition of the metric $g_{\mu \nu}[9,10]$,

$g_{00}=-N^{2}+N_{i} h^{i j} N_{j}, \quad g_{0 i}=N_{i}, \quad g_{i j}=h_{i j}$,

$g^{00}=-\frac{1}{N^{2}}, \quad g^{0 i}=\frac{N^{i}}{N^{2}}, \quad g^{i j}=h^{i j}-\frac{N^{i} N^{j}}{N^{2}}$,

where we have defined $h^{i j}$ as the inverse of the induced metric $h_{i j}$ on the Cauchy surface $\Sigma_{t}$ at each time $t$,

$$
h_{i k} h^{k j}=\delta_{i}^{j},
$$

and we denote $N^{i}=h^{i j} N_{j}$. The four-dimensional scalar curvature in the $3+1$ formalism has the form

$$
\begin{aligned}
R\left(g_{\mu \nu}\right)= & K_{i j} \mathcal{G}^{i j k l} K_{k l}+{ }^{(3)} R+\frac{2}{\sqrt{-g}} \partial_{\mu}\left(\sqrt{-g} n^{\mu} K\right) \\
& -\frac{2}{\sqrt{h} N} \partial_{i}\left(\sqrt{h} h^{i j} \partial_{j} N\right) \\
\equiv & K_{i j} \mathcal{G}^{i j k l} K_{k l}+{ }^{(3)} R+\Xi
\end{aligned}
$$

where the extrinsic curvature of the spatial hypersurface $\Sigma_{t}$ at the time $t$ is defined as

\footnotetext{
${ }^{2}$ For a review of cosmological fluctuations, see for example Ref. [11].
}

PHYSICAL REVIEW D 95, 084009 (2017)

$$
K_{i j}=\frac{1}{2 N}\left(\frac{\partial h_{i j}}{\partial t}-D_{i} N_{j}-D_{j} N_{i}\right)
$$

with $D_{i}$ being the covariant derivative determined by the metric $h_{i j}$ and where the de Witt metric is defined as

$$
\mathcal{G}^{i j k l}=\frac{1}{2}\left(h^{i k} h^{j l}+h^{i l} h^{j k}\right)-h^{i j} h^{k l}
$$

with inverse

$$
\mathcal{G}_{i j k l}=\frac{1}{2}\left(h_{i k} h_{j l}+h_{i l} h_{j k}\right)-\frac{1}{2} h_{i j} h_{k l},
$$

which obeys the relation

$$
\mathcal{G}_{i j k l} \mathcal{G}^{k l m n}=\frac{1}{2}\left(\delta_{i}^{m} \delta_{j}^{n}+\delta_{i}^{n} \delta_{j}^{m}\right)
$$

Further, $n^{\mu}$ is the future-pointing unit normal vector to the hypersurface $\Sigma_{t}$, which is written in terms of the ArnowittDeser-Misner (ADM) variables as

$$
n^{0}=\sqrt{-g^{00}}=\frac{1}{N}, \quad n^{i}=-\frac{g^{0 i}}{\sqrt{-g^{00}}}=-\frac{N^{i}}{N} .
$$

In order to formulate the restricted $f(R)$ gravity, we break the full diffeomorphism invariance of the $f(R)$ gravity by performing the replacement

$$
R \rightarrow R+(\Upsilon-1) \Xi
$$

where $\Upsilon$ is a dimensionless parameter that controls the breaking of the diffeomorphism invariance of the action. Even if the shift (10) seems to be very mild, it turns out that it has a significant impact on the Hamiltonian structure of this theory as was shown in Ref. [8]. Careful analysis performed there showed that in order to have a consistent theory from the Hamiltonian analysis point of view, it is necessary to include terms which depend on the spatial derivative of the lapse $a_{i}=\frac{\partial_{i} N}{N}$ into the action. Further, the breaking of the diffeomorphism invariance suggests the possibility to define the theory with the generalized de Witt metric $[13,14]$

$$
\begin{aligned}
& \tilde{\mathcal{G}}^{i j k l}=\frac{1}{2}\left(h^{i k} h^{j l}+h^{i l} h^{j k}\right)-\lambda h^{i j} h^{k l}, \quad \lambda \neq \frac{1}{3}, \\
& \tilde{\mathcal{G}}_{i j k l}=\frac{1}{2}\left(h_{i k} h_{j l}+h_{i l} h_{j k}\right)-\frac{\lambda}{3 \lambda-1} h_{i j} h_{k l} .
\end{aligned}
$$

In summary, we proposed in Ref. [8] the extended form of the restricted $f(R)$ gravity when we performed the replacement 


$$
\begin{aligned}
R \rightarrow R_{\Upsilon} \equiv & K_{i j} \tilde{\mathcal{G}}^{i j k l} K_{k l}+{ }^{(3)} R+\Upsilon \Xi+\gamma_{1} a_{i} a^{i} \\
& +\gamma_{2}{ }^{(3)} R^{i j} a_{i} a_{j},
\end{aligned}
$$

where $\gamma_{1}, \gamma_{2}$ are the corresponding coupling constants. We showed there that this theory is consistent from the Hamiltonian analysis point of view since the structure of constraints is the same as in the case of nonprojectable $f(R)$ Hořava-Lifshitz gravity [15-17]; for the Hamiltonian analysis, see Refs. [18-21] and also Refs. [22-27]. Explicitly, we showed that there is no Hamiltonian first class constraint, but it is a second class constraint together with momentum conjugate to lapse $N$. This Hamiltonian constraint can be solved for $N$ at least in principle, which is fundamentally different from the case of full diffeomorphism-invariant $f(R)$ gravity where $N$ can be considered a free parameter. Then, due to the restricted number of constraints, the number of physical degrees of freedom is higher than in the case of $f(R)$ gravity, and it is equal to the number of degrees of freedom in nonprojectable $f(R)$ Hořava-Lifshitz gravity. We also showed that this theory has two global first class constraints that reflect an invariance of the theory under foliation preserving diffemorphism [14]. In summary, restricted $f(R)$ gravity has a very rich Hamiltonian structure and can be considered a very nice model of theories with restricted diffeomorphism invariance. Moreover, we also showed that it has also very interesting cosmological solutions that we briefly review in the next section.

\section{BACKGROUND EQUATIONS}

In this section, we derive the equations of motion for the restricted $f(R)$ gravity when we presume the FRW form of the background. Recall that the restricted $f\left(R / M_{P}^{2}\right)$-gravity action has the form

$S_{\text {res }}=\int d^{4} x \sqrt{-g}\left[M_{P}^{2} \frac{R_{\Upsilon}}{2}+\eta M_{P}^{4} f\left(\frac{R_{\Upsilon}}{M_{P}^{2}}\right)\right]+S_{\text {Matter }}$,

where $R_{\Upsilon}$ is defined in (12). The matter part contributes to the gravitational equations of motion through the stressenergy tensor which is defined as

$$
T_{\mu \nu}=-\frac{2}{\sqrt{-g}} \frac{\delta S_{\text {Matter }}}{\delta g^{\mu \nu}}
$$

Our goal is to analyze the spatially homogeneous and isotropic Universe so that the metric ansatz is the FRW metric which has the form

$$
d s^{2}=-N(t)^{2} d t^{2}+a(t)^{2} d x^{i} d x^{j} \delta_{i j},
$$

where $N=N(t)$ is the lapse and $a=a(t)$ is the scale factor. As usual, the Hubble parameter is defined as
$H \equiv \dot{a} / a$. Note that we cannot set $N(t)=1$ from the beginning due to the restricted form of the diffeomorphism. But, since for the background equations all quantities depend only on time, one can use the redefinition of time in (15) to set $N=1$ in the background equations. We also presume matter in the form of a prefect fluid, which means that the stress-energy tensor has the form

$$
T_{\beta}^{\alpha}=\left(\begin{array}{cccc}
-\rho(t) & 0 & 0 & 0 \\
0 & p(t) & 0 & 0 \\
0 & 0 & p(t) & 0 \\
0 & 0 & 0 & p(t)
\end{array}\right) .
$$

Since we presume that the full diffeomorphism is broken in the gravitational sector only, we find that the matter action is diffeomorphism invariant. As a result, the stress-energy tensor is conserved in the sense $\nabla_{\mu} T^{\mu \nu}=0$ on the condition that the matter satisfies the equation of motion. Now, for the background (15), we obtain

$\Gamma_{00}^{0}=\frac{\dot{N}}{N}, \quad \Gamma_{i j}^{0}=\frac{1}{N^{2}} \dot{a} a \delta_{i j}, \quad \Gamma_{0 i}^{0}=0$,

$\Gamma_{00}^{i}=0, \quad \Gamma_{j 0}^{i}=\delta_{j}^{i} H, \quad \Gamma_{j k}^{i}=0$

so that the conservation of the stress-energy tensor implies the standard conservation equation

$$
\dot{\rho}+3 H(\rho+p)=0 .
$$

Using (15) and the general relations in the ADM formalism, which are discussed in Ref. [10], one can show that

$$
\Xi=-6 \frac{H \dot{N}}{N^{3}}+6 \frac{\dot{H}}{N^{2}}+18 \frac{H^{2}}{N^{2}} .
$$

The generalized Friedmann equation for the model has been determined in Ref. [8] as

$$
3 A H^{2}+\eta M_{P}^{2} f+\eta\left(6 A H^{2}-R_{\Upsilon}\right) F+6 \eta \Upsilon H \dot{F}=\frac{\rho}{M_{P}^{2}},
$$

where

$$
\begin{aligned}
R_{\Upsilon} & =-6 A H^{2}+\Upsilon\left(6 \dot{H}+18 H^{2}\right), \quad f \equiv f\left(\frac{R_{\Upsilon}}{M_{P}^{2}}\right), \\
F & \equiv M_{P}^{2} \frac{d f}{d R_{\Upsilon}} \equiv f^{\prime}, \quad A \equiv \frac{3 \lambda-1}{2} .
\end{aligned}
$$

Note that we have defined the prime as a derivative with respect to the argument of $f$. As a result, $F, f^{\prime \prime}, \ldots$ are dimensionless quantities. Finally, we note that there is still another equation after performing the time derivative of Eq. (20) and then use Eq. (19), 
$A \dot{H}(1+2 \eta F)+\eta H(2 A-3 \Upsilon) \dot{F}+\Upsilon \eta \ddot{F}=-\frac{(\rho+p)}{2 M_{P}^{2}}$.

After this brief review of the background equations, we now switch to the main topic of this paper which is the analysis of the cosmological perturbations.

\section{COSMOLOGICAL PERTURBATIONS}

The goal of this section is to derive equations for the perturbed FRW space-time in the ADM formalism. ${ }^{3}$ As a check of the validity of our approach, we note that for $\Upsilon=$ $1, \lambda=1, \gamma_{1}$ and $\gamma_{2}=0$ the results derived in this paper should reduce to the corresponding results of the standard $f\left(R / M_{P}^{2}\right)$ gravity; see for example the review [5].

To begin with, we emphasize that we are writing our equations in the Newtonian gauge which is defined by $N^{i}=0$. We use the Newtonian gauge for the following reason: As it has been argued in Ref. [11], one of the advantages of the Newtonian gauge is that the physical fields which are defined by the gauge fixing coincide with the gauge-invariant variables. Note also that it is an easy task to move from the Newtonian gauge to other gauges [11]. Finally, note that, due to the fact that the spatial section of the metric (15) is flat, it is natural to use the Fourier decomposition of the perturbation where the Fourier components of a general perturbation $U(t, \mathbf{x})$ are defined as

$$
U_{k}(t)=\int d^{3} \mathbf{x} U(t, \mathbf{x}) e^{-i \mathbf{k} \cdot \mathbf{x}},
$$

where $\mathbf{x}=\left(x^{1}, x^{2}, x^{3}\right), \mathbf{k}=\left(k^{1}, k^{2}, k^{3}\right)$. Further, we also decompose $F$ and $\dot{F}$ into the homogeneous and perturbed parts as

$$
F=\bar{F}+\delta F, \quad \dot{F}=\dot{\bar{F}}+\dot{\delta F},
$$

where - over any quantity shows the unperturbed part of that quantity. In the case of the fluctuations of the matter, we use the following parametrization for the perturbed energy-momentum tensor

$$
\delta T_{0}^{0}=-\delta \rho, \quad \delta T_{0}^{i}=-(\rho+p) \partial_{i} v, \quad \delta T_{i}^{j}=\delta p \delta_{i}^{j},
$$

where $v$ is the potential for the spatial velocity of the fluid.

\footnotetext{
${ }^{3}$ For a review, see Ref. [10].
}

\section{A. Scalar metric perturbations}

Before we proceed to the study of the perturbed equations, it is instructive to discuss the role of diffeomorphism symmetry in the cosmological context.

For a full diffeomorphism-invariant model, as for example the Einstein-Hilbert action or $f(R)$-gravity action, there is no a priori preferred coordinate system. Of course, the symmetry can be broken by imposing other restrictions on the model. For example, in the cosmological context, it has been shown that the only coordinate system (observer) that is compatible with the assumption of homogeneity and isotropy of space is described by the FRW metric [28]. The uniqueness of the FRW metric helps us to choose it as the background metric for our model in this paper. But the uniqueness of the metric is broken if we consider a universe which is not homogeneous. The deviation from homogeneity is considered as the perturbed FRW metric. For example, the scalar metric perturbations can be parametrized as [11]

$$
\begin{aligned}
d s^{2}= & -(1+2 Y) d t^{2}+2 a \partial_{i} B d x^{i} d t+a^{2}\left[(1+2 \zeta) \delta_{i j}\right. \\
& \left.+2 \partial_{i} \partial_{j} E\right] d x^{i} d x^{j},
\end{aligned}
$$

where $Y=Y(t, \mathbf{x}), B=B(t, \mathbf{x}), E=E(t, \mathbf{x}), \zeta=\zeta(t, \mathbf{x})$ are 3 -scalars.

It is an unnecessary and senseless task to insert the above expression into general equations of motion in order to obtain the equations of motion for these 3 -scalars. The reason for this statement is that, similar to the model with the full diffeomorphism symmetry, here we confront with the so-called gauge problem. The problem arises when we note that the model is invariant under the coordinate transformation as $t \rightarrow t+P(t), x^{i} \rightarrow x^{i}+\partial^{i} K(t, \mathbf{x})$, where $\partial^{i}=\delta^{i j} \partial_{j}$. Under this transformation, the components of the metric (26) transform as [11]

$$
\begin{aligned}
Y \rightarrow Y^{t} & =Y-\dot{P}(t), \\
B & \rightarrow B^{t}=B+\frac{P(t)}{a}-a \dot{K}(t, \mathbf{x}), \\
E \rightarrow E^{t} & =E-K(t, \mathbf{x}), \\
\zeta & \rightarrow \zeta^{t}=\zeta-H P(t) .
\end{aligned}
$$

Therefore, even if we tried to obtain the equations for the components of Eq. (26), we would have some solutions which are unphysical in the sense that they can be derived by the application of the transformation (27) on some particular solutions. In order to avoid the above problem, i.e. the gauge problem, one can choose a specific gauge (coordinate) or use the gauge-invariant quantities. In the process of the gauge fixing, values for $P(t)$ and $K(t, \mathbf{x})$ are explicitly specified such that we have not any residual symmetry for the solution. Physically, gauge fixing means that a specific spatial coordinate has been chosen. 
In the following, we will work with the Newtonian gauge. The Newtonian gauge is defined as the perturbed metric for observers who are immobile at the hypersurfaces of constant time. Also, the normal vector of the hypersurfaces is the same as the worldlines of the observers at any time. Thus, the Newtonian gauge is described by [11]

$$
d s^{2}=-(1+2 Y) d t^{2}+a^{2}\left[(1+2 \zeta) \delta_{i j}\right] d x^{i} d x^{j} .
$$

The Newtonian gauge is the preferred gauge for the latetime cosmology as was argued in Ref. [3]. Therefore, we will use it to compare the results of our model with the Plank observations. Finally, we should emphasize that it is still possible to construct the gauge-invariant quantities from the Newtonian gauge variables [11]. To proceed further, we note that it is convenient to parametrize the scalar metric perturbations in the Newtonian gauge as

$$
\begin{aligned}
& N^{2}=1+2 Y \equiv e^{2 \Phi}, \\
& h_{i j}=a^{2}(1+2 \zeta) \delta_{i j} \equiv a^{2} e^{-2 \Psi} \delta_{i j},
\end{aligned}
$$

where $\Phi$ and $\Psi$ are space and time dependent. Using these definitions in (12), we easily find

$$
\begin{aligned}
\left.R_{\Upsilon}\right|_{\text {scalar }}= & { }^{3} R+6(3 \Upsilon-A) e^{-2 \Phi}(H-\dot{\Psi})^{2} \\
& +6 \Upsilon e^{-2 \Phi}(\dot{H}-\ddot{\Psi})-6 \Upsilon \dot{\Phi} e^{-2 \Phi}(H-\dot{\Psi}) \\
& -2 \Upsilon \frac{e^{2 \Psi}}{a^{2}} \partial^{2} \Phi+2 \Upsilon \frac{e^{2 \Psi}}{a^{2}} \partial_{i} \Phi \partial_{i} \Psi \\
& +\left(\gamma_{1}-2 \Upsilon\right) \frac{e^{2 \Psi}}{a^{2}} \partial_{i} \Phi \partial_{i} \Phi .
\end{aligned}
$$

As a check, we note that for $\Phi=\Psi=0$ the above relation reduces to the background form of $R_{\Upsilon}$ given in Eq. (21). Now, by performing the linearization of the expression (30), we obtain the Fourier component of $\left.\delta R_{\Upsilon}\right|_{\text {scalar }}$,

$$
\begin{aligned}
\left.\delta R_{\Upsilon}\right|_{\text {scalar }}(k)= & \frac{-4 k^{2}}{a^{2}} \Psi+\frac{2 \Upsilon k^{2}}{a^{2}} \Phi-12(3 \Upsilon-A) H^{2} \Phi \\
& -12 \Upsilon \dot{H} \Phi-6 \Upsilon H \dot{\Phi}-6 \Upsilon \ddot{\Psi} \\
& -12(3 \Upsilon-1) H \dot{\Psi},
\end{aligned}
$$

where $k^{2} \equiv k_{i} k^{i}$. Further, inserting (25) and (29) into Eq. (18) and by performing the corresponding linearization, we obtain two equations,

$$
\dot{\delta \rho}+3 H(\delta \rho+\delta p)=\frac{k^{2}}{a^{2}} \delta q+3(\rho+p) \dot{\Psi},
$$

and

$$
\dot{\delta q}+3 H \delta q+\delta p+(\rho+p) \Phi=0,
$$

where $\delta q \equiv-(\rho+p) v$. Note that these equations have the same forms as the corresponding equations for the usual $f\left(R / M^{2}\right)$ gravity. In order to derive the remaining equations, we proceed in the following way. To begin with, we insert (30) into the action. As we pointed out, we work in the Newtonian gauge. Then, in order to vary the action with respect to the shift, it is sufficient to consider the terms which are proportional to $N_{i} \Psi$ and $N_{i} \Phi$. For example, if we define $\delta_{N_{i}}$ as a variation with respect to the shift, then for $S_{\text {Matter }}$, we obtain

$$
\begin{aligned}
\delta_{N_{i}} S_{\text {Matter }} & =-\frac{1}{2} \int d^{4} x \sqrt{-g} T^{\mu \nu} \delta_{N_{i}} g_{\mu \nu} \\
& =-\frac{1}{2} \int d^{4} x a^{3} T^{0 i} \delta N_{i}+\mathcal{O}\left(N_{i}^{2}\right) .
\end{aligned}
$$

Using this result and also using the relations

$$
n^{\mu}=\left(e^{-\Phi},-N^{i} e^{-\Phi}\right), \quad \Gamma_{i j}^{i}=-3 \partial_{j} \Psi
$$

and after some integration by parts, we obtain

$$
\begin{aligned}
S= & -A M_{P}^{2} \int d^{4} x 2 a[H \Phi+\dot{\Psi}] \partial_{i} N_{i}(1+2 \eta \bar{F}) \\
& -4 A \eta M_{P}^{2} \int d^{4} x a H N_{i} \partial_{i} \delta F-2 \eta \Upsilon M_{P}^{2} \int d^{4} x a\left[N_{i} \partial_{i} \dot{\delta F}\right. \\
& \left.-\partial_{i} \Phi N_{i} \dot{\bar{F}}-3 H N_{i} \partial_{i} \delta F\right]+S_{\text {Matter }}
\end{aligned}
$$

Further on, performing the variation with respect to $N_{i}$ and using the Fourier components of the perturbations, we obtain

$$
\begin{aligned}
A(H \Phi+\dot{\Psi})(1+2 \eta \bar{F})= & \eta[\Upsilon \dot{\delta F}-\Upsilon \Phi \dot{\bar{F}}+(2 A-3 \Upsilon) H \delta F] \\
& -\frac{1}{2 M_{P}^{2}} \delta q .
\end{aligned}
$$

In the case of the variation of the action with respect to $\Phi$, we set $N_{i}=0$ in the action and then expand the action up to the second order in $\Phi$ and $\Psi$. This procedure, some integration by parts and using Eq. (20) leads to

$$
\begin{aligned}
\delta_{\Phi} S_{\text {res }}= & \int d^{4} x a^{3} M_{P}^{2} \delta \Phi\left[\left(-12 A \Phi H^{2}+\frac{4}{a^{2}} \partial^{2} \Psi\right.\right. \\
& \left.-12 A H \dot{\Psi}-\frac{2 \gamma_{1}}{a^{2}} \partial^{2} \Phi\right) \times\left(\frac{1}{2}+\eta \bar{F}\right) \\
& +\eta\left((12 A-18 \Upsilon) H^{2}-6 \Upsilon \dot{H}-\frac{2 \Upsilon}{a^{2}} \partial^{2}\right) \delta F \\
& \left.-\eta \dot{\bar{F}}(12 \Upsilon H \Phi+6 \Upsilon \dot{\Psi})+6 \eta \Upsilon H \dot{\delta} \dot{F}-\delta_{\Phi} S_{\text {Matter }}\right]
\end{aligned}
$$


so that we easily find the Fourier form of the equation of motion for $\Phi$,

$$
\begin{aligned}
& \eta\left[(6 A-9 \Upsilon) H^{2}-3 \Upsilon \dot{H}+\frac{\Upsilon}{a^{2}} k^{2}\right] \delta F-3 \Upsilon \eta \dot{\bar{F}}(2 H \Phi+\dot{\Psi})+3 \eta \Upsilon H \dot{H} \bar{F}-\frac{\delta \rho}{2 M_{P}^{2}} \\
& \quad=\left(3 A \Phi H^{2}+\frac{k^{2}}{a^{2}} \Psi+3 A H \dot{\Psi}-\gamma_{1} \frac{k^{2}}{2 a^{2}} \Phi\right)(1+2 \eta \bar{F}) .
\end{aligned}
$$

For reasons that will become clear later, we derive $\delta p$ in two different ways. In the first case, we use (32) and (39). Then, in order to eliminate $(\rho+p)$ in these formulas, we use (22). Collecting these pieces together, we obtain an equation

$$
\begin{aligned}
\frac{\delta p}{2 M_{P}^{2}}= & \eta\left(2 A \dot{\Psi}+4 A H \Phi+\Upsilon \dot{\Phi}-\gamma_{1} \frac{k^{2}}{3 H a^{2}} \Phi\right) \dot{\bar{F}}-2 \eta H \dot{\delta}+2 \eta \Upsilon \Phi \ddot{\bar{F}}-\eta \Upsilon \ddot{\delta} \ddot{F}+\eta\left[(3 \Upsilon-2 A) \dot{H}+(9 \Upsilon-6 A) H^{2}\right. \\
& \left.+\frac{2 k^{2}}{3 a^{2}}(A-2 \Upsilon)\right] \delta F+\left[\dot{\Phi} H+2 A \dot{H} \Phi+A \ddot{\Psi}+3 A \Phi H^{2}+3 A H \dot{\Psi}+\frac{k^{2}}{3 a^{2}}(\Psi-A \Phi)+(1-A) \frac{k^{2}}{3 H a^{2}} \dot{\Psi}\right. \\
& \left.-\gamma_{1} \frac{k^{2}}{6 a^{2}}\left(\frac{\dot{\Phi}}{H \Phi}+1\right) \Phi\right](1+2 \eta \bar{F}),
\end{aligned}
$$

where we also used the fact that $\dot{R}_{\Upsilon} \delta F=\delta R_{\Upsilon} \dot{\bar{F}}$.

The second way to derive the relation for $\delta p$ is to use Eqs. (33) and (37). We again use Eq. (22) in order to eliminate $(\rho+p)$ so that we obtain

$$
\begin{aligned}
\frac{\delta p}{2 M_{P}^{2}}= & \eta[2 \dot{\Psi}+2(1+A) H \Phi+\Upsilon \dot{\Phi}] \dot{\bar{F}}-2 \eta A H \dot{\delta} \dot{F}+2 \eta \Upsilon \Phi \ddot{\bar{F}}-\eta \Upsilon \dot{\delta F}+\eta\left[(3 \Upsilon-2 A) \dot{H}+(9 \Upsilon-6 A) H^{2}\right] \delta F \\
& +\left[\dot{\Phi} H+2 \dot{H} \Phi+\ddot{\Psi}+3 \Phi H^{2}+3 H \dot{\Psi}\right](1+2 \eta \bar{F})
\end{aligned}
$$

The right-hand sides of Eqs. (40) and (41) are the same if

$$
\begin{aligned}
& {\left[(1-A) \dot{\Phi} H+(1-A) \frac{k^{2}}{3 H a^{2}} \dot{\Psi}+\frac{k^{2}}{3 a^{2}}(\Psi-A \Phi)-\frac{\gamma_{1} k^{2}}{6 a^{2}}\left(\frac{\dot{\Phi}}{H \Phi}+1\right) \Phi\right][1+2 \eta \bar{F}]} \\
& \quad=2 \eta \frac{k^{2}}{3 a^{2}}(2 \Upsilon-A) \delta F+\eta\left[2(1-A) H \Phi+2(1-A) \dot{\Psi}+\gamma_{1} \frac{k^{2}}{3 H a^{2}} \Phi\right] \dot{\bar{F}} .
\end{aligned}
$$

As is clear, the equations are simplified by taking $\lambda=1$, which results in $A=1$. As a check, note that for $\Upsilon=1, \lambda=1$ and $\gamma_{1}=0$ these equations match the corresponding relations for the usual $f\left(R / M_{P}^{2}\right)$ gravity.

\section{B. Tensor metric perturbations}

In this section, we focus on the metric tensor perturbations $\gamma_{i j}$. Recall that the perturbed line element has the form

$$
d s^{2}=-d t^{2}+a^{2}\left[\delta_{i j}+\gamma_{i j}\right] d x^{i} d x^{j}
$$

where $\partial_{i} \gamma_{i j}=\gamma_{i}^{i}=0$. In order to derive equations in this sector, we use the fact that $\sqrt{-g}$ does not contain the metric tensor perturbations up to the second order. Further, using the traceless condition on $\gamma_{i j}$, it is easy to show that the metric perturbations do not appear in $K$. Thus, it turns out that the terms in (12) which are proportional to $\Upsilon$ and $\lambda$ do not contribute to the metric tensor perturbations. As a result, the analysis of this sector is very similar to the analysis of tensor fluctuations in standard $f(R)$ gravity. Explicitly, the second order action for these modes has the form

$$
\left.\delta S\right|_{\text {tensor }}=\frac{M_{P}^{2}}{8} \int d^{4} x[1+2 \eta \bar{F}]\left[a \gamma_{i j} \partial^{2} \gamma_{i j}+a^{3} \dot{\gamma}_{i j}^{2}\right]
$$

Then, in order to avoid the ghost instability, we must impose the following condition:

$$
1+2 \eta \bar{F}>0
$$

Performing a variation of (44) with respect to $\gamma_{i j}$ and using the Fourier representation 


$$
\gamma_{i j}=\int \frac{d^{3} k}{(2 \pi)^{3 / 2}} \sum_{s= \pm} \epsilon_{i j}^{s}(k) \gamma_{k}^{s}(t) e^{i \vec{k} . \vec{x}},
$$

where $\epsilon_{i i}=k^{i} \epsilon_{i j}=0$ and $\epsilon_{i j}^{s}(k) \epsilon_{i j}^{s^{\prime}}(k)=2 \delta_{s s^{\prime}}$, leads to the second order differential equation for $\gamma_{k}^{s}$,

$$
\ddot{\gamma_{k}^{s}}+\dot{\gamma}_{k}^{s} \frac{d}{d t} \ln \left[a^{3}(1+2 \eta \bar{F})\right]+\left(\frac{k}{a}\right)^{2} \gamma_{k}^{s}=0,
$$

which has the same form as in the standard $f(R)$ gravity.

\section{Vector metric perturbations}

In this section, we perform an analysis of vector metric perturbations. As in the case of the prefect fluid, we consider the following form of the perturbed stress tensor in the vector sector

$$
\left.\delta T_{i}^{0}\right|_{\text {vector }}=\delta q_{i}^{V} .
$$

Then, from $\nabla_{\mu} T^{\mu \nu}=0$, it follows that

$$
\delta \dot{q}_{i}^{V}+3 H \delta q_{i}^{V}=0 .
$$

As in the case of the metric perturbations, the favorite gauge in this sector is the so-called vector gauge which is defined by

$$
d s^{2}=-d t^{2}+2 a S_{i} d x^{i} d t+a^{2} \delta_{i j} d x^{i} d x^{j},
$$

where $\partial_{i} S_{i}=0$. Again, from the above definition and the condition on $S_{i}$, it turns out that the terms in (13) which are proportional to $\Upsilon$ do not contribute in this sector. Then, the second order action takes the following form:

$$
\begin{aligned}
\left.\delta S\right|_{\text {vector }}= & -\frac{M_{P}^{2}}{2} \int d^{4} x a S_{i} \partial^{2} S_{i}(1+2 \eta \bar{F}) \\
& -2 \int d^{4} x a^{2} S_{i} \delta q_{i}^{V} .
\end{aligned}
$$

Then, it is easy to see that the equation for $S_{i}$ in the Fourier space has the form

$$
M_{P}^{2} k^{2}(1+2 \eta \bar{F}) \frac{S_{i}}{a}=2 \delta q_{i}^{V}
$$

which coincides with the equation for the vector perturbation in the standard $f(R)$ gravity.

\section{SOME CONSTRAINTS ON THE PARAMETERS}

In this section, we explore some constraints on the parameters of the model. We will focus on cases for which the model can be solved analytically. So, we can compare our results with the Einstein-Hilbert model. In this section, we take $A=1$ that corresponds to $\lambda=1$. Note that this is a natural presumption, since it is expected that $\lambda$ should approach 1 in the low energy regime [13,14]. It is important to emphasize that the Einstein-Hilbert gravity predicts $\Phi=\Psi$. In the more general case when $\Phi \neq \Psi$, it is useful to define an anisotropic stress, $\delta \Sigma$, as

$$
\Psi-\Phi=8 \pi G a^{2} \delta \Sigma .
$$

Clearly, a nonvanishing anisotropic stress is the signature of modification of general relativity $[3,12]$. Further, it is also convenient to define

$$
\gamma \equiv \frac{\Psi}{\Phi}
$$

so that the Einstein-Hilbert gravity predicts $\gamma=1$. But from the Planck cosmic microwave background (CMB) temperature data, the current value for $\gamma$ is reported as [3]

$$
\gamma_{0}-1=0.70 \pm 0.94
$$

which is shown by yellow and gray colors in Fig. 1. On the other hand, if we consider the weak lensing data, we have [3]

$$
\gamma_{0}-1=1.36_{-0.69}^{+1.0}
$$

which is shown by the gray and blue colors in Fig. 1.

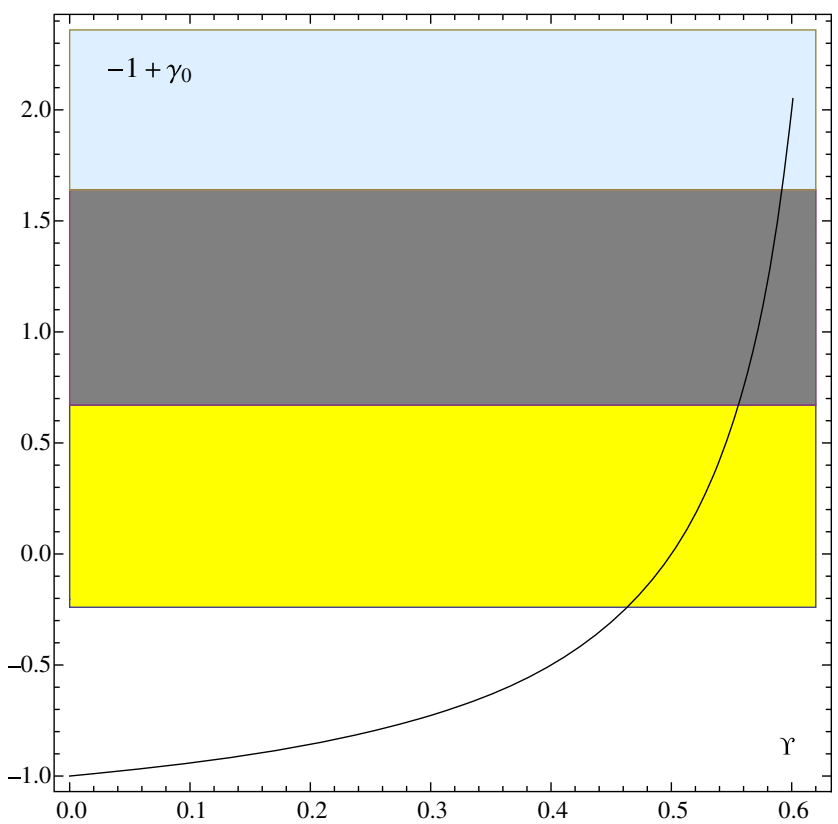

FIG. 1. Space of parameters $\left(\gamma_{0}-1, \Upsilon\right)$ for the model. The yellow and gray regions show the values for $\gamma_{0}-1$ from the Planck CMB temperature data [3]. The gray and blue regions show the values for $\gamma_{0}-1$ from the combination of the Planck CMB temperature data and the weak lensing [3]. The solid line represents the prediction of the model in the de Sitter space for $\gamma_{1} \ll 1$ 
Let us now return to our model and consider the late-time cosmology when we can neglect the radiation and the cold dark matter. Explicitly, we consider two cases:

(i) Case I.-In this case, we presume that the cosmological constant $\Lambda$ is nonzero while $\eta=0$.

The energy density of the cosmological constant is $\rho=\Lambda M_{P}^{2}$ so that (20) implies

$$
H^{2}=\frac{\Lambda}{3} \text {. }
$$

Note that this result is the same as in the case of Einstein-Hilbert gravity with the cosmological constant.

Since for the cosmological constant we have $\delta q=\delta p=\delta \rho=0$, from Eqs. (37) and (42), we have

$$
\begin{aligned}
\dot{\Psi}+H \Phi & =0, \\
\Psi-\Phi & =\frac{\gamma_{1}}{2}\left(\frac{\dot{\Phi}}{H \Phi}+1\right) \Phi .
\end{aligned}
$$

If we combine these two equations together, we obtain

$$
\ddot{\Psi}+\left(\frac{2}{\gamma_{1}}+1\right) H \dot{\Psi}+\frac{2}{\gamma_{1}} H^{2} \Psi=0
$$

that has the solution

$$
\begin{gathered}
\Psi=C_{1} e^{-H t}+C_{2} e^{-\frac{2}{\gamma_{1}} H t} \quad\left(\text { for } \gamma_{1} \neq 2\right), \\
\Psi=C_{1} e^{-H t}+C_{2} H t e^{-H t} \quad\left(\text { for } \gamma_{1}=2\right),
\end{gathered}
$$

where $C_{1}$ and $C_{2}$ are arbitrary constants. It is clear that in order to avoid instability we have to require that $\gamma_{1}>0$. Using now Eqs. (54), (58) and (60), we obtain

$\gamma-1=\frac{\gamma_{1}}{2}\left[1-\frac{C_{1} e^{-H t}+C_{2} \frac{4}{\gamma_{1}^{2}} e^{-\frac{2}{\gamma_{1}} H t}}{C_{1} e^{-H t}+C_{2} \frac{2}{\gamma_{1}} e^{-\frac{2}{\gamma_{1}} H t}}\right] \quad\left(\right.$ for $\left.\gamma_{1} \neq 2\right)$

and

$$
\gamma-1=\frac{C_{2}}{C_{1}-C_{2}+C_{2} H t} \quad\left(\text { for } \gamma_{1}=2\right) .
$$

For the present Universe, we have $H_{0} t_{0} \approx 1$, and it is easy to see that we can choose the parameters in the above equations to obtain a consistent result with the reported data for $\gamma_{0}-1$. Note also that the above relations show that if we take $t \rightarrow \infty$ we have

$$
\begin{aligned}
& \gamma \rightarrow 1, \quad \text { if } \gamma_{1} \leq 2, \\
& \gamma \rightarrow \frac{\gamma_{1}}{2}, \quad \text { if } \gamma_{1}>2 .
\end{aligned}
$$

To gain insight into the effect of the model in structure formation, let us study the matterdominated era for which $p=\delta p=0$. To obtain an analytical solution and compare it with the Einstein-Hilbert case, let us consider $f=0$ in our formalism. Again, in this case, the background equations are the same as the Einstein-Hilbert. So, we have $H=\frac{2}{3 t}$. From Eqs. (41) and (42), we have

$$
\begin{gathered}
\frac{\delta p}{2 M_{P}^{2}}=\dot{\Phi} H+2 \dot{H} \Phi+\ddot{\Psi}+3 \Phi H^{2}+3 H \dot{\Psi} . \\
\Psi=\Phi\left(1+\frac{\gamma_{1}}{2}\right)+\frac{\gamma_{1}}{2} \frac{\dot{\Phi}}{H} .
\end{gathered}
$$

By eliminating $\Psi$ from Eq. (65) and Eq. (66), we have

$$
\frac{\gamma_{1}}{2 H} \dddot{\Phi}+\left(1+\frac{7}{2} \gamma_{1}\right) \ddot{\Phi}+\left(4+\frac{15}{4} \gamma_{1}\right) H \dot{\Phi}=0 .
$$

The above equation has power-low solutions as $\Phi \propto t^{n}$ for which $n$ is given by

$$
\begin{aligned}
& 3 \frac{\gamma_{1}}{24} n(n-1)(n-2)+\left(1+\frac{7}{2} \gamma_{1}\right) n(n-1) \\
& +\left(4+\frac{15}{4} \gamma_{1}\right) \frac{2}{3} n=0 .
\end{aligned}
$$

For $\gamma_{1}=0$ the Eq. (68) has two solutions. The first one is $n=0$ that corresponds to the static gravitational potential. The second solution is $n=\frac{-5}{3}$ that corresponds to the decaying mode that can be neglected in the study of the structure formation. Finally for $\gamma_{1} \neq 0$ the Eq. (68) has following equations

$$
n=0, \quad n=\frac{-4-5 \gamma_{1} \pm \sqrt{\Delta}}{6 \gamma_{1}}
$$

where

$$
\Delta=16-40 \gamma_{1}+\gamma_{1}^{2} .
$$

Note that, compared with the Einstein-Hilbert case, we have an additional solution for $\Phi$, which is the consequence of the model. The condition for $\Delta>0$ can be obtained by 


$$
\begin{aligned}
& \gamma_{1} \leq 20-8 \sqrt{6} \approx 0.4, \\
& \gamma_{1} \geq 20+8 \sqrt{6} \approx 40 .
\end{aligned}
$$

As is clear, in both cases, we have $n<0$, and we can neglect them in the study of structure formation.

(ii) Case II.-In the second case, we neglect the cosmological constant, but $\eta \neq 0$ so that we have only two independent equations which determine $\Phi$ and $\Psi$. One of these equations is obtained by multiplying Eq. (37) with $3 H$ and then using the corresponding result to eliminate some terms in Eq. (39). This procedure gives the following equation (for $A=1$ ):

$$
\begin{aligned}
& {\left[\frac{2 k^{2}}{a^{2}} \Psi-\gamma_{1} \frac{k^{2}}{a^{2}} \Phi\right](1+2 \eta \bar{F})} \\
& \quad=-6 \eta \Upsilon[\dot{\Psi}+H \Phi] \dot{\bar{F}}+6 \eta \Upsilon\left(\frac{k^{2}}{3 a^{2}}-\dot{H}\right) \delta F
\end{aligned}
$$

Further, Eq. (42) takes the following form for $A=1$ :

$$
\begin{aligned}
& {\left[(\Psi-\Phi)-\frac{\gamma_{1}}{2}\left(\frac{\dot{\Phi}}{H \Phi}+1\right) \Phi\right][1+2 \eta \bar{F}]} \\
& \quad=2 \eta(2 \Upsilon-1) \delta F+\frac{\eta \gamma_{1}}{H} \Phi \dot{\bar{F}} .
\end{aligned}
$$

In the de Sitter space, which is a very good approximation for the late-time cosmology, Eqs. (72) and (73) simplify considerably:

$$
\left[2 \Psi-\gamma_{1} \Phi\right](1+2 \eta \bar{F})=2 \eta \Upsilon \delta F
$$

and

$$
\begin{aligned}
& {\left[(\Psi-\Phi)-\frac{\gamma_{1}}{2}\left(\frac{\dot{\Phi}}{H \Phi}+1\right) \Phi\right][1+2 \eta \bar{F}]} \\
& \quad=2 \eta(2 \Upsilon-1) \delta F
\end{aligned}
$$

If we combine these two equations, we obtain the following results:

$$
\begin{aligned}
2(3 \Upsilon & -2) \Psi \\
= & \Upsilon \gamma_{1}\left(3-\frac{\dot{\Phi}}{H \Phi}\right) \Phi-2\left(\Upsilon+\gamma_{1}\right) \\
& \times \Phi\left(\text { for } \Upsilon \neq 0, \Upsilon \neq \frac{1}{2}\right), \\
\Psi= & \frac{\gamma_{1}}{2} \Phi \quad(\text { for } \Upsilon=0),
\end{aligned}
$$

$$
(\Psi-\Phi)=\frac{\gamma_{1}}{2}\left(\frac{\dot{\Phi}}{H \Phi}+1\right) \Phi, \quad\left(\text { for } \Upsilon=\frac{1}{2}\right)
$$

Note that for $\Upsilon=2 / 3$ the first equation implies

$$
\frac{\dot{\Phi}}{H \Phi}=-\frac{2}{\gamma_{1}} . \quad\left(\text { for } \Upsilon=\frac{2}{3}\right)
$$

Finally, when we insert (76) and (77) into Eq. (74) or Eq. (75), we obtain the equations for the remaining variables that can be easily solved. Equations (76) are interesting results since they give a relation between the parameters of the model and observation. If we take the $\gamma_{1} \ll 1$ limit, it follows that

$$
\begin{aligned}
(3 \Upsilon-2) \Psi & \approx-\Upsilon \Phi \quad\left(\text { for } \Upsilon \neq 0, \frac{1}{2}, \frac{2}{3}\right), \\
\frac{\Psi}{\Phi} & \ll 1 \quad(\text { for } \Upsilon=0), \\
\frac{\Psi}{\Phi} & \approx 1 \quad\left(\text { for } \Upsilon=\frac{1}{2}\right) .
\end{aligned}
$$

Note that, except for $\Upsilon=2 / 3$, in this limit (i.e. $\left.\gamma_{1} \ll 1\right)$, the above relations do not depend on the form and dynamics of $f$ in the action (13). As shown in Fig. 1, for $\gamma_{1} \ll 1$, one can choose $\Upsilon$ to reconcile the model with the current observations.

\section{CONCLUSION}

This short paper is devoted to the analysis of the cosmological fluctuations of the restricted $f(R)$ theory of gravity. We determined the background equations of motion and then carefully analyzed the fluctuations above this background solution. We show that the vector and tensor fluctuations have the same dynamics as in the case of the standard $f(R)$ gravity, while the scalar sector possesses new interesting possibilities which depend on the values of coupling constants. In more details, we show that it is possible to choose the values of these parameters so that the predictions of the restricted $f(R)$ gravity are in agreement with recent observation data. In fact, we showed previously in Ref. [8] that the cosmological solutions found in the restricted $f(R)$ gravity are in agreement with observation, and our current analysis of fluctuations confirms this fact as well. This is by itself an interesting result that suggests that it is indeed plausible to consider theories with the restricted diffeomorphism invariance as interesting alternatives to the fully diffeomorphism-invariant theories of gravity.

\section{ACKNOWLEDGMENTS}

The work of J. K. was supported by the Grant Agency of the Czech Republic under Grant No. P201/12/G028. 
[1] P. A. R. Ade et al. (Planck Collaboration), Planck 2015 results. XX. Constraints on inflation, Astron. Astrophys. 594, A20 (2016).

[2] A. A. Starobinsky, A new type of isotropic cosmological models without singularity, Phys. Lett. B 91, 99 (1980).

[3] P. A. R. Ade et al. (Planck Collaboration), Planck 2015 results. XIV. Dark energy and modified gravity, Astron. Astrophys. 594, A14 (2016).

[4] S. Weinberg, The cosmological constant problem, Rev. Mod. Phys. 61, 1 (1989).

[5] A. De Felice and S. Tsujikawa, $\mathrm{f}(\mathrm{R})$ theories, Living Rev. Relativ. 13, 3 (2010).

[6] N. Arkani-Hamed, P. Creminelli, S. Mukohyama, and M. Zald arriaga, Ghost inflation, J. Cosmol. Astropart. Phys. 04 (2004) 001.

[7] C. Cheung, P. Creminelli, A. Liam Fitzpatrick, J. Kaplan, and L. Senatore, The effective field theory of inflation, J. High Energy Phys. 03 (2008) 014.

[8] M. Chaichian, A. Ghalee, and J. Kluson, Restricted f(R) gravity and its cosmological implications, Phys. Rev. D 93, 104020 (2016).

[9] R. L. Arnowitt, S. Deser, and C. W. Misner, The dynamics of general relativity, Gen. Relativ. Gravit. 40, 1997 (2008).

[10] E. Gourgoulhon, 3+1 Formalism and Bases of Numerical Relativity, arXiv:gr-qc/0703035.

[11] V. F. Mukhanov, H. A. Feldman, and R. H. Brandenberger, Theory of cosmological perturbations, Phys. Rep. 215, 203 (1992).

[12] E. Di Valentino, A. Melchiorri, and J. Silk, Cosmological hints of modified gravity?, Phys. Rev. D 93, 023513 (2016).

[13] P. Horava, Membranes at quantum criticality, J. High Energy Phys. 03 (2009) 020.

[14] P. Horava, Quantum gravity at a Lifshitz point, Phys. Rev. D 79, 084008 (2009).

[15] D. Blas, O. Pujolas, and S. Sibiryakov, Consistent Extension of Horava Gravity, Phys. Rev. Lett. 104, 181302 (2010).

[16] D. Blas, O. Pujolas, and S. Sibiryakov, Models of nonrelativistic quantum gravity: The good, the bad and the healthy, J. High Energy Phys. 04 (2011) 018.
[17] D. Blas, O. Pujolas, and S. Sibiryakov, Comment on "Strong coupling in extended Horava-Lifshitz gravity", Phys. Lett. B 688, 350 (2010).

[18] J. Kluson, Note about Hamiltonian formalism of healthy extended Horava-Lifshitz gravity, J. High Energy Phys. 07 (2010) 038.

[19] W. Donnelly and T. Jacobson, Hamiltonian structure of Horava gravity, Phys. Rev. D 84, 104019 (2011).

[20] S. Mukohyama, R. Namba, R. Saitou, and Y. Watanabe, Hamiltonian analysis of nonprojectable Hooava-Lifshitz gravity with $U(1)$ symmetry, Phys. Rev. D 92, 024005 (2015).

[21] M. Chaichian, J. Kluson, and M. Oksanen, Nonprojectable Horava-Lifshitz gravity without the unwanted scalar graviton, Phys. Rev. D 92, 104043 (2015).

[22] J. Kluson, Horava-Lifshitz f(R) Gravity, J. High Energy Phys. 11 (2009) 078.

[23] J. Kluson, S. Nojiri, S. D. Odintsov, and D. Saez-Gomez, U (1) invariant $F(R)$ Horava-Lifshitz gravity, Eur. Phys. J. C 71, 1690 (2011).

[24] J. Kluson, Note about Hamiltonian formalism of modified $F(R)$ Hořava-Lifshitz gravities and their healthy extension, Phys. Rev. D 82, 044004 (2010).

[25] J. Kluson, New models of $f(R)$ theories of gravity, Phys. Rev. D 81, 064028 (2010).

[26] S. Carloni, M. Chaichian, S. Nojiri, S. D. Odintsov, M. Oksanen, and A. Tureanu, Modified first-order HoravaLifshitz gravity: Hamiltonian analysis of the general theory and accelerating FRW cosmology in power-law $\mathrm{F}(\mathrm{R})$ model, Phys. Rev. D 82, 065020 (2010); Errattum, Phys. Rev. D 85, 129904(E) (2012).

[27] M. Chaichian, S. Nojiri, S. D. Odintsov, M. Oksanen, and A. Tureanu, Modified F(R) Horava-Lifshitz gravity: A way to accelerating FRW cosmology, Classical Quantum Gravity 27, 185021 (2010); Errattum, Classical Quantum Gravity 29, 159501 (2012).

[28] S. Weinberg, Gravitation and Cosmology (Wiley, New York, 1972). 